\title{
A Three-pronged View on Organizational
}

\section{Agility}

\author{
IT Professional (forthcoming)
}

Karl Werder *

Cologne Institute for Information Systems, University of Cologne, Germany

Phil Hennel

Cologne Institute for Information Systems, University of Cologne, Germany

Mareike Fischer

AXA Konzern AG, Cologne, Germany and Cologne Institute for Information Systems, University of Cologne, Germany
Janek Richter

Cologne Institute for Information Systems, University of Cologne, Germany

Tim Dreesen

Cologne Institute for Information Systems, University of Cologne, Germany

Janina Weingarth

Comea, Frankfurt, Germany and Cologne Institute for Information Systems, University of Cologne, Germany

* (corresponding author) 


\title{
A Three-pronged View on Organizational
}

\section{Agility}

\begin{abstract}
The ability of organizations to sense and respond to changes-defined as organizational agilityis considered by senior executives among their top information technology (IT) concerns as an important ability for organizations on their quest toward sustained competitive advantage. However, every transformation toward agility also comes at a cost, requiring resource commitment and IT landscape changes. We present examples of successful and unsuccessful attempts at achieving agility while leveraging IT. Our presented cases focus on information systems development agility, customer agility, and entrepreneurial agility. Our findings suggest that agility is neither achieved easily, nor is a guarantor for success. Depending on the context and implementation of organizational agility, however, it can significantly improve process and product performance. We develop a three-pronged view consisting of a functional, temporal, and ambidextrous view to resolve these challenges. We end with three recommendations for practitioners that seek to shape their organization's journey toward agility.
\end{abstract}

\section{Keywords}

D.2.9.e Organizational management and coordination; K.4.3 Organizational Impacts

\section{Motivation}

Markets characterized by uncertainty and turbulence have become the norm raising the need for the ability of organizations to seize opportunities and respond to threats with ease, speed, and dexterity (e.g. [1]). Market dynamics, mergers and acquisitions, new product requirements, and technological innovation are only few examples of environmental changes organizations face. The ability of organizations to sense and respond to changes - defined as organizational agility [2] - is a top information technology (IT) concerns among senior executives since the beginning of the last decade [3]. Agility is an important ability for 
organizations on their quest toward sustained competitive advantage and generally improves organizational performance (e.g., [4]).

While organizations facing continuous and unforeseen change often see agility as a silver bullet that solves all problems, such organizations tend to ignore the implications of becoming agile. Every transformation toward agility also comes at a cost [5], as it requires resource commitment and changes to the IT landscape. Furthermore, there are industries such as the medical industry with their highly reliable organizations in which core principles of agility are difficult to implement: for example, medical products cannot be developed in small batches and adapted based on customer feedback. Instead, strict regulations and the necessity to be fail-proof require such organizations to finalize development before releasing a product to the market.

\section{Stories from the Field}

In the following, we present examples of successful and unsuccessful attempts at achieving agility while leveraging IT. We focus on selected cases from industry that showcase information systems development (ISD) agility, customer agility, and entrepreneurial agility. ISD agility enables development teams to cope with rapidly changing environments, customer agility facilitates co-opting customers in the exploration and exploitation of opportunities, and entrepreneurial agility enables the organization to anticipate and seize market opportunities proactively.

\section{A Digital-Native Company: ISD Agility at Spotify}

Spotify is native to digital product development and innovation and is the biggest on-demand music streaming platform worldwide by numbers of subscribers. Its service reached over 100 million subscribers and 217 million monthly active users in 2019 [6]. Based on a freemium business model, the offerings include a basic service freely available and an advertise-free, high-quality subscription. In literature, the company's organizational model is famously referred to as the 'Spotify-Model' (e.g., [7]) and has sparked interest among the consolidators and proponents of governance models for implementing scaled agile.

The Spotify-Model originated from a publication by Spotify itself based on a blog entry of two of its employees, in which they lay out how Spotify organizes its product development [7]. Spotify managed to scale agile practices beyond 30 development teams in various locations 
and established an agile mindset within the organization. Central to their matrix model is the division of the organization into feature- or functionality-oriented vs. skill-based organizational entities. The former are the so-called Squads, which represent the basic development units toward a specific product functionality (similar to the Scrum team and staffed with a product owner who is responsible for work prioritization). The latter are represented by so-called "Chapters" and "Guilds" that combine employees with a similar set of skills and competencies (Chapter) or a common interest (Guilds; similar to a community of interest).

Considering a matrix organization, Spotify's organizational design creates a healthy tension between 'what' should be developed (ideally a great product) and 'how' it should be developed (ideally by technical excellence). The company attributes its steady growth rate along a high degree of employee satisfaction to its organizational design choices [7].

\section{A Transformation Story: Customer Agility at LEGO}

LEGO shows how agility can be expanded outside of the context of software development and in combination with traditional stage-gate models that serve the development of physical goods. LEGO-founded in 1932-is still seeking to "inspire and develop the builders of tomorrow" with more than 17,000 employees in 15 countries [8]. The organization's structure is a wheel, suggesting their flat hierarchy and reduction of silos. The company maintains a collaborative and fun culture and embraces innovation.

While the company strives today, it was on the verge to collapse in 2004. LEGO recovered by focusing on its core products and customer-centric values, following the company's motto "only the best is good enough" [8]. In 2011, the company launched an innovative education initiative that led to a new product within 12 months. Resulting from the integration of software developers that were familiar with "being agile", their team playfully explored the adoption of agile practices in a heavily stage-gate driven environment [9]. The results were admirable, as both speed and customer interaction increased which ultimately led to increased project performance. Following the positive experience in the pilot project, the company expanded the rollout of agile practices within the entire organization.

The adoption of agile practices within the project helped the LEGO team to improve communication through daily stand-up meetings, visual scrum boards, daily activity logs and prioritized project backlogs [9]. As a result, misunderstandings decreased, and the overall 
productivity increased. Furthermore, LEGO developed a platform for digital customer engagement. The team also reported improved workflows and confidence in problem solving abilities through the involvement of the entire team during the next stand-up.

\section{The Decline of an Empire: Lack of Entrepreneurial Agility at Nokia}

Nokia is a cautionary tale about how agility in itself is not a guarantee for success but a capability that has to be built up and orchestrated across organization, processes, technology, and culture. In early 2008, Nokia mobile phones were ubiquitous, and its global market share had reached a peak at $39 \%$. Only 5 years later, with a mere remaining global market share of 14\%, Nokia announced the sale of its devices and service business to Microsoft [10]. How was this demise possible for a successful company, known for its entrepreneurial agility? Nokia initially faced the downturn in the early 2000s successfully by restructuring its organization several times and by institutionalizing an evolutionary new-venture strategy process. This agile management philosophy later materialized in constant changes of organizational structure and management team composition [10].

However, informal organizational processes increased dramatically as a consequence, bringing decision processes to a near halt and increasing internal competition for shared resources to destructive levels [10]. Furthermore, Nokia's mobile phone operating system (OS) Symbian did not have a modular architecture and was tightly coupled with a multitude of devices resulting in a technical disadvantage of their products. While efficient for the lowcost market, Nokia's technology was not set-up to compete with the disruptive ecosystem approach from iOS and Android. Several parallel attempts to develop an alternative OS did not succeed. Neither did the later conversion of Symbian OS to open source as well as the switch to the Microsoft OS in 2011 turn out to be successful. The two OS competitors iOS and Android were already too well established on the market.

The situation was further aggravated with middle manager fear and rivalry, stifling internal politics and deteriorating technological competence on top management level. Nokia unfortunately lost its way toward entrepreneurial agility and technological leadership to renew itself. At this point in time, visionary strategic choices and routinized technology management could have been the solution [10]. 


\section{Achieving Agility through IT}

Agility-as a concept, method, or as an organizational trait-is neither achieved easily, nor a guarantor for success as the case of Nokia shows. Depending on the context and implementation it can, however, improve process and product performance significantly, given the adequate structures as described in the case of Spotify. It can, as seen in the case of LEGO, radically change organizational culture and processes. These cases suggest: the adoption of agile practices may help the organization to develop a form of agility (e.g., ISD agility, entrepreneurial agility) which may only reflect one specific view on organizational agility. Research has used cases such as these three to study the preconditions and effects of agility in different contexts, environments, and implementations. While research on concepts of agility is still ongoing and organizations still fail reaping the benefits of agility, we discuss the role of IT and present the overarching findings of current research.

The pivotal role of IT is not only seen in practice, but also reflects recent research interests [4]. Prior studies suggest that IT-enabled agile capabilities of the organization are especially important in highly turbulent environments [11]. This applies to transformative cases, as the example of LEGO shows, and digital native cases, as for example the case of Spotify suggests. Both cases exemplify the importance of IT capabilities when establishing organizational agility. IT can help the organization to respond to environmental changes. For example, increased flexibility of the IT system has positive effects toward agility and organizational performance, especially in more turbulent environments [12]. When extending IT flexibility with IT standardization or IT alignment, scholars use the term IT ambidexterity, i.e., the achievement of two seemingly conflicting goals. Organizations can utilize IT ambidexterity in order to increase their organization's level of agility, especially in dynamic environments [13].

When taking a more nuanced perspective toward organizational agility, prior research distinguishes for example between entrepreneurial agility (proactive changes) and adaptive agility (reactive changes) [1]. IT competences help organizations to achieve entrepreneurial agility especially in more dynamic environments. Larger organizations - such as the cases described above - benefit from their organizational size for their sensing capabilities [14], IT architecture modularity, and IT governance decentralization, when establishing organizational agility. Neglecting the importance of IT can have severe consequences, as the case of Nokia suggests. In sum, research suggests that there is no normative one size fits all approach to 
agility; rather, it is a multi-dimensional and context-sensitive capability that should be shaped according to the specific company situation.

\section{The State-of-the-Art in Research}

Conceptual ambiguity still challenges the clear understanding of the concept and its boundaries. From an academic perspective, the literature offers us various conceptualizations for agility. Focusing on an ISD perspective, agility is defined as the combination of flexibility and leanness resulting in the continual readiness of an ISD method [15]. A dualistic perspective is taken when dividing agility into entrepreneurial agility and adaptive agility [1]. While the former takes a proactive and anticipating stance, the latter has a reactive focus to implement changes after they occurred. Finally, a threefold perspective is taken when delineating agility into operational, customer, and partnering agility [16]. See Table 1 for an overview of these conceptualizations in literature.

Table 1. Exemplary Concepts of Organizational Agility

\begin{tabular}{|l|l|}
\hline Concept & Definition \\
\hline ISD Agility & $\begin{array}{l}\text { The continual readiness of an ISD method to rapidly or inherently create } \\
\text { change, proactively or reactively embrace change, and learn from change } \\
\text { while contributing to perceived customer value (economy, quality, and } \\
\text { simplicity), through its collective components and relationships with its } \\
\text { environment. [15] }\end{array}$ \\
\hline Adaptive Agility & $\begin{array}{l}\text { The firm detects and responds to market dynamics in a defensive manner, } \\
\text { such as protecting itself and remaining resilient, generally in an attempt to } \\
\text { recover from disruptions in market forces rather than in response to any } \\
\text { fundamental change in the internal structure or organization. [1] }\end{array}$ \\
Agility & $\begin{array}{l}\text { Ability to anticipate and seize market opportunities proactively and thus } \\
\text { allows a firm to modify its positioning and strategies and organize new } \\
\text { business approaches to gain early advantages in changing conditions. [1] }\end{array}$ \\
\hline Customer Agility & $\begin{array}{l}\text { The co-opting of customers in the exploration and exploitation of } \\
\text { opportunities for innovation and competitive action moves. [16] }\end{array}$
\end{tabular}




\begin{tabular}{|l|l|}
$\begin{array}{l}\text { Operational } \\
\text { Agility }\end{array}$ & $\begin{array}{l}\text { The ability of firms' business processes to accomplish speed, accuracy, and } \\
\text { cost economy in the exploitation of opportunities for innovation and } \\
\text { competitive action. [16] }\end{array}$ \\
Partnering & $\begin{array}{l}\text { The ability to leverage the assets, knowledge, and competencies of } \\
\text { suppliers, distributors, contract manufacturers, and logistics providers } \\
\text { Agility }\end{array}$
\end{tabular}

These three conceptualizations focus on different domains around the organization. Some forms of agility are rather outward directed (e.g., partnering agility and entrepreneurial agility), while others focus on internals of the organization (e.g., ISD agility and operational agility). Figure 1 provides an overview of the connection between different agility conceptualizations, the organization, and its environment.

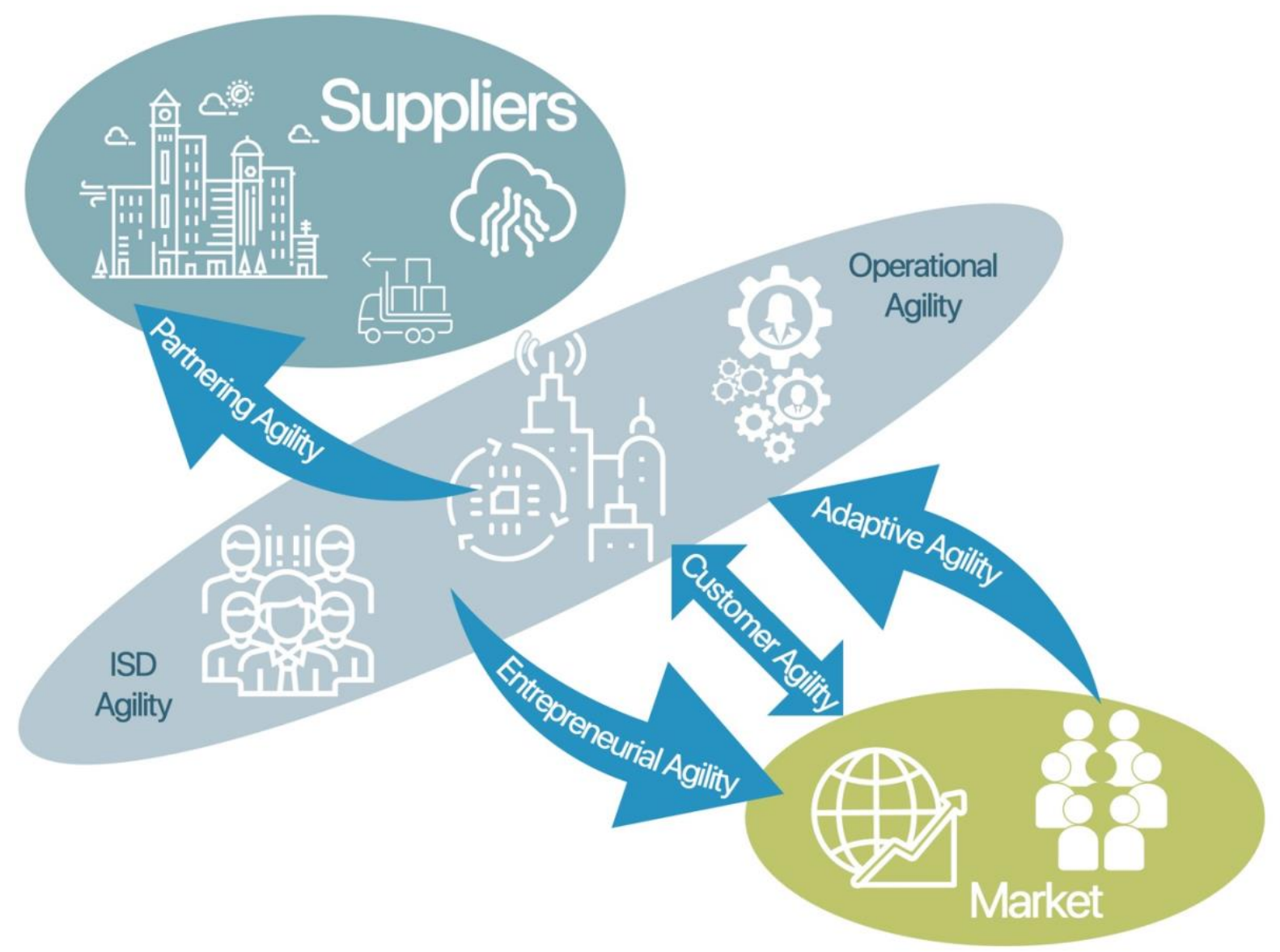

Figure 1. Different Concepts of Organizational Agility 


\section{A Three-pronged View on Organizational Agility}

Organizational agility can take various forms and is a context-sensitive organizational capability. As such, organizations face three challenges when becoming agile: cost of transformation, different speeds of adoption, and intra-organizational tensions. First, building up IT capabilities to support organizational agility requires financial investments on top of the already incurred costs of an agile transformation. Second, larger organizations may also face challenges from different speeds of adoption of its organizational units. While organizational functions that employ software developers may already be familiar with the idea, other functions may start adopting agile practices from scratch, which also require adjustments to the practices themselves (cf. LEGO case). Third, tensions within the organization arise when sub-units set different foci, e.g., focusing on sensing the environment and being proactive versus focusing on responding and being reactive to environmental changes.

In response to these challenges, we develop an integrative framework toward organizational agility based on three dominating views. First, a functional view investigates performance improvements resulting from agility (e.g., [12], [16]). Second, a temporal view investigates the mechanisms behind an initial sensing and subsequent responding toward environmental changes (e.g., [2]). Third, an ambidextrous view sheds light onto tensions that may arise (e.g., [1], [13]). We discuss each view and its resolution to the above challenges in turn.

The functional view describes agility as an organizational ability that mediates the effect of IT toward performance implications. Examples include the effects of IT infrastructure toward improving customer agility [17], the effects of business process agility toward organizational performance [18], or the effect of marketing agility toward financial performance. Focusing on functional elements of organizational agility - e.g., customers, operations, partners - helps organizations to identify and assign a value to the costs they have invested. IT investments for increasing sensing and responding capability in functional areas might seem like additional costs of agility. However, increasing agility is often only the motivation for an investment into IT capabilities that is required in any case to remain competitive. Evaluation measures can include reduced cycle times, increased customer satisfaction, or number of competitive actions that help the organization to understand the value they receive for the dollars they spend. For example, Spotify was able to outperform its competitors through their ability to quickly respond during product development. 
The temporal view of agility focuses on the sensing and responding components of agility [2], [17]. Here, environmental changes cause different episodic events that an organization needs to sense and respond to [2]. The organizational challenge is to manage the unpredictable changes in their environment and to harness them for achieving organizational goals [19]. In the temporal view, IT is deeply embedded (and not perceived as a separate enabler) in a continuous process perspective. Taking a temporal view helps organizations clearly identify sequences in which they progress when transforming to an agile organization. Achieving agility is not a one-off conversion but a process that requires continuous reevaluation, learning, and acting (each of which inextricably linked with IT). For example, first, organizations adopt agile practices and deploy corresponding roles. Only thereafter, can they work towards benefiting from an agile mindset through increased autonomy and selforganization. For example, Lego needed some time to understand, adjust and adopt different agile practices before employees benefitted from increased and open communication. This was only possible through a continuous sensing and responding process.

For the ambidextrous view, different tensions have been identified. For example, proactive versus reactive stance or planned dynamic capability versus improvisational capability. A proactively agile organization is able to identify and act upon market opportunities as soon as they arise and quickly build up competitive advantages from changing environments (e.g., [1], [2], [16]), whereas a reactively agile organization is resilient to external changes and adapts and transforms itself with ease and speed (e.g., [1]). An improvisational, agile organization can spontaneously react to unprecedented environmental changes and quickly reconfigure its resources (e.g., [19]), while a planned-dynamically agile organization is able to exploit windows of opportunity through structured, planned, and disciplined capability building processes (e.g., [13]).

The ambidextrous view helps organizations to clearly identify conflicting objectives so that they can be balanced, adopting one of two responding tactics: integration or differentiation. Organizations may integrate the conflicting objectives within the same unit so that a balance can be achieved. Alternatively, they may actively separate them so that two different units achieve one of these objectives individually; yet, the overarching organization achieves both. In this view, the role of IT is twofold, either actively driving innovation or stabilizing the organization in times of turbulence. Thanks to their agile model, Spotify's developing teams 
for example strike the balance between developing a technology-driven innovative product that services current customer demands while assuring operational excellence.

The COVID-19 pandemic has shown that organizational agility is more important for organizational survival than ever before. While some companies have been able to quickly shift their business model-e.g., Louis Vuitton quickly shifted their production from luxury products toward essential nonsurgical facemasks-others struggled while hoping for subsidies by local governments-e.g., Volkswagen, Daimler and BMW demanded publicly funded rebates. From here on, we suggest extending agility research and practices with the ability to absorb shocks and keep fundamental values stable. While an organization's ability to sense and respond to changes is advantageous, avoiding too frequent or unnecessary changes can be beneficial when changes are temporary. For example, LEGO revitalized their long-standing mission of operational excellence, stating that "only the best is good enough". Even with their environment, technologies, and markets changed, LEGO's core mission remained the same.

\section{Conclusion}

Organizational agility equips organizations with the ability to react to changes in order to survive in today's cut-throat competitive environment. We presented three cases from industry, focusing on ISD agility, customer agility, and entrepreneurial agility. We suggest that IT plays an important role in establishing organizational agility that fits the organizational culture and processes. Our review of the literature on organizational agility lead us to propose a three-pronged view toward organizational agility: i) a functional view that investigates IT capability as antecedents of agility; ii) a temporal view that investigates an embedded role of IT in sequencing of sensing and responding capabilities; iii) and an ambidextrous view that suggests a dialectic perspective toward IT to "exploit old certainties" and "explore new possibilities" [20].

IT practitioners need to get involved in the business strategy formulation to unleash the full potential of the organization's IT and increase IT-driven innovation on the organization's journey toward agility. In sum, we derive the following recommendations for IT professionals:

- First, build proactive IT capability to assist your organization in sensing its environment, technological advances and market trends. Usage of IT needs to be engrained in the organizations business planning activities, spanning all functions. 
- Second, harness reactive IT capability, helping your organization to improve its ongoing operations and processes. Building on the proactive IT capability, reactive IT capability helps your organization to implement new processes, technologies, and business models.

- Third, start with building proactive IT capability followed by reactive IT capability. Despite this sequential implementation, professionals need to assure that the organization strikes a balance and does not neglect one capability over the other.

\section{References}

[1] A. Chakravarty, R. Grewal, and V. Sambamurthy, "Information Technology Competencies, Organizational Agility, and Firm Performance: Enabling and Facilitating Roles," Inf. Syst. Res., vol. 24, no. 4, pp. 976-997, Dec. 2013.

[2] E. Overby, A. Bharadwaj, and V. Sambamurthy, "Enterprise agility and the enabling role of information technology," Eur. J. Inf. Syst., vol. 15, no. 2, pp. 120-131, Apr. 2006.

[3] L. Kappelman, R. Torres, E. McLean, C. Maurer, V. Johnson, and K. Kim, "The 2018 SIM IT Issues and Trends Study," MIS Q. Exec., vol. 18, no. 1, p. Article 7, 2019.

[4] P. P. Tallon, M. Queiroz, T. Coltman, and R. Sharma, "Information technology and the search for organizational agility: A systematic review with future research possibilities," J. Strateg. Inf. Syst., vol. 28, no. 2, pp. 218-237, Jun. 2019.

[5] D. Teece, M. Peteraf, and S. Leih, "Dynamic capabilities and organizational agility: Risk, uncertainty, and strategy in the innovation economy," Calif. Manage. Rev., vol. 58, no. 4, pp. 13-35, Aug. 2016.

[6] Spotify USA Inc., "Spotify Reports First Quarter 2019 Earnings," FINANCIAL PERFORMANCE, 2019. [Online]. Available: https://newsroom.spotify.com/2019-0429/spotify-reports-first-quarter-2019-earnings/. [Accessed: 14-Oct-2019].

[7] H. Kniberg and A. Ivarsson, "Scaling Agile @ Spotify - with Tribes, Squads, Chapters \& Guilds." Spotify Technology S.A., Stockholm, SE, p. 14, 2012.

[8] Lego Group, “THE LEGO GROUP CULTURE,” Lego.com US, 2019. [Online]. Available: https://www.lego.com/en-us/careers/culture. [Accessed: 30-Sep-2019].

[9] R. G. Cooper and A. F. Sommer, "The Agile-Stage-Gate Hybrid Model: A Promising 
New Approach and a New Research Opportunity," J. Prod. Innov. Manag., vol. 33, no. 5, pp. 513-526, Sep. 2016.

[10] J.-A. Lamberg, S. Lubinaitè, J. Ojala, and H. Tikkanen, "The curse of agility: The Nokia Corporation and the loss of market dominance in mobile phones, 2003-2013," Bus. Hist., pp. 1-47, Jun. 2019.

[11] P. A. Pavlou and O. A. El Sawy, "The 'Third Hand': IT-Enabled Competitive Advantage in Turbulence Through Improvisational Capabilities," Inf. Syst. Res., vol. 21, no. 3, pp. 443-471, Sep. 2010.

[12] P. P. Tallon and A. Pinsonneault, "Competing Perspectives on the Link Between Strategic Information Technology Alignment and Organizational Agility: Insights from a Mediation Model," MIS Q., vol. 35, no. 2, p. 463, 2011.

[13] O.-K. (Daniel) Lee, V. Sambamurthy, K. H. Lim, and K. K. Wei, "How Does IT Ambidexterity Impact Organizational Agility?," Inf. Syst. Res., vol. 26, no. 2, pp. 398417, Jun. 2015.

[14] Y. Park, O. A. El Sawy, and P. Fiss, "The Role of Business Intelligence and Communication Technologies in Organizational Agility: A Configurational Approach," J. Assoc. Inf. Syst., vol. 18, no. 9, pp. 648-686, 2017.

[15] K. Conboy, "Agility from First Principles: Reconstructing the Concept of Agility in Information Systems Development," Inf. Syst. Res., vol. 20, no. 3, pp. 329-354, Sep. 2009.

[16] Sambamurthy, Bharadwaj, and Grover, "Shaping Agility through Digital Options: Reconceptualizing the Role of Information Technology in Contemporary Firms," MIS Q., vol. 27, no. 2, p. 237, 2003.

[17] N. Roberts and V. Grover, "Investigating firm's customer agility and firm performance: The importance of aligning sense and respond capabilities," J. Bus. Res., vol. 65, no. 5, pp. 579-585, May 2012.

[18] Y. Chen, Y. Wang, S. Nevo, J. Jin, L. Wang, and W. S. Chow, "IT capability and organizational performance: the roles of business process agility and environmental factors," Eur. J. Inf. Syst., vol. 23, no. 3, pp. 326-342, May 2014.

[19] M. van Oosterhout, E. Waarts, and J. van Hillegersberg, "Change factors requiring agility and implications for IT," Eur. J. Inf. Syst., vol. 15, no. 2, pp. 132-145, Apr. 2006.

[20] J. G. March, "Exploration and Exploitation in Organizational Learning," Organ. Sci., vol. 
2, no. 1, pp. 71-87, Feb. 1991. 